\title{
Development of Biosensor based on Imaging Ellipsometry and its Applications
}

\author{
Gang Jin ${ }^{\mathrm{a}}$ \\ ${ }^{a}$ NML, Institute of Mechanics, Chinese Academy of Sciences, \#15 Bei-si-huan west Rd., \\ Beijing 100190, P.R. China
}

\begin{abstract}
We have reviewed the development of the biosensor based on imaging ellipsometry including its principle, methodology and general engineering model structure, mainly compared experimental setups between the previous one and the recently developed one. It's obvious that the sensitivity and the signal to noise ratio has been improved by a various spectroscopic light source, the optimization of polarized components setting and a cool CCD, especially the contribution of the CCD, which makes the biosensor available in more and more biomedical applications.
\end{abstract}

Keywords: Biosensor, imaging ellipsometry, protein interaction, protein microarray.

\section{INTRODUCTION}

The concept of biosensor based on imaging ellipsometry was proposed 15 years ago [1]. The prototype of experimental setup and the evaluation on the biosensor application was successively reported [2]. It has been developed into an automatic analysis technique for protein detection with merits of label-free, quantitative, high throughput and real-time analysis for protein interaction process, etc. Protein delivery and interaction is performed with a micro-fluidic reactor array. Imaging ellipsometry is used to visualize the molecule surface concentration distribution of protein microarray on a solid substrate. The protein microarray is designed upon the demand of target detection, such as the target molecule type, concentration, numbers and detection conditions. The biosensor is based on biomolecule specific affinity that one reactant as a ligand is immobilized to a surface to form a monolayer as a bioprobe to recognize its receptor. With the visualization of imaging ellipsometry which has a high spatial resolution in the order of $0.1 \mathrm{~nm}$ in vertical and micron in lateral, the complex of ligand-receptor can be determined, and in this way, the existence of the specific biomolecule to the ligand can be verified. Many bioprobes arrayed in matrix that is a protein microarray are used for high throughput protein detection. Its principle, methodology, related technique units, biosensor system and previous works are reviewed in Ref. [3].

With the development of its engineering model and applications, especially biomedical applications, further demand on the sensitivity and flexibility of the biosensor is more important. The flexibility mainly depends on the mechanical, electrical, informatics and biological control. To the requirement in biomedical applications, its operational mechanism and the signal to noise ratio (SNR) could not be fitted, especially for cases in the real-time analysis of protein interaction and protein weak interaction, so the biosensor in total internal reflection (TIR) mode of imaging ellipsometry [4] has been developed. It combines the microfluidic reactor array with imaging ellipsometer for an in-situ system. Considering the dispersive effect of the microarray, the related experimental condition to the sensitivity and SNR, we have introduced a spectroscopic light source, optimization settings of polarization components and a low noise CCD imaging which brings an obvious improvement in the sensitivity and SNR. It makes specifications of the biosensor more fitted to practical requirements, the sensitivity for immunoglobulin molecule in $1 \mathrm{ng} / \mathrm{mL}$, the consumption of ligand in ng and specimen in the order of $10 \mu \mathrm{l}$, the throughput of 48 independent channels, and the time resolution of $0.04 \mathrm{~s}$ as well as a continues variable spectrum in visible region. These parameters do not reach to their limitations and could be further improved upon requirements.

In order to show an overview of the biosensor, we look back the principle, methodology and the experimental setup; especially pay attention to the imaging ellipsometer and its improvement in this report.

Sensors, Cameras, and Systems for Industrial, Scientific, and Consumer Applications XII,

edited by Ralf Widenhorn, Valérie Nguyen, Proc. of SPIE-IS\&T Electronic Imaging, SPIE Vol. 7875

78750E · @ 2011 SPIE-IS\&T · CCC code: 0277-786X/11/\$18 - doi: 10.1117/12.871981

Proc. of SPIE-IS\&T/ Vol. 7875 78750E-1 


\section{PRINCIPLE AND METHOD [3]}

Imaging ellipsometry is used to visualize the molecule mass surface concentration distribution of protein layers attached on a patterned surface as shown in Figure 1. A ligand and its receptor such as an antibody and its corresponding antigen could assemble into bio-complexes due to their specific affinity. The optical biosensor is based on that each reactant as a ligand is immobilized to a surface to form a monolayer as a bioprobe with its bioactivity. The other reactant as the analyte (or receptor) existed in a solution. The bioprobe is exposed to the solution containing analyte. When the analyte in the solution interacts with its corresponding ligand on the bioprobe and assembled into complex upon their affinity. The molecule mass surface concentration on the surface where the interaction takes place becomes higher than before exposure to the analyte solution. A significant increase of the surface concentration indicates that the solution contains receptor against the ligand on the surface. With the visualization of imaging ellipsometry which has a high spatial resolution in the order of $0.1 \mathrm{~nm}$ in vertical and micron in lateral, the increase could be determined, and in this way, the existence of the analyte in the solution can be verified. Many bioprobes arrayed in matrix to form a protein microarray is used for high throughput detection [5-7].

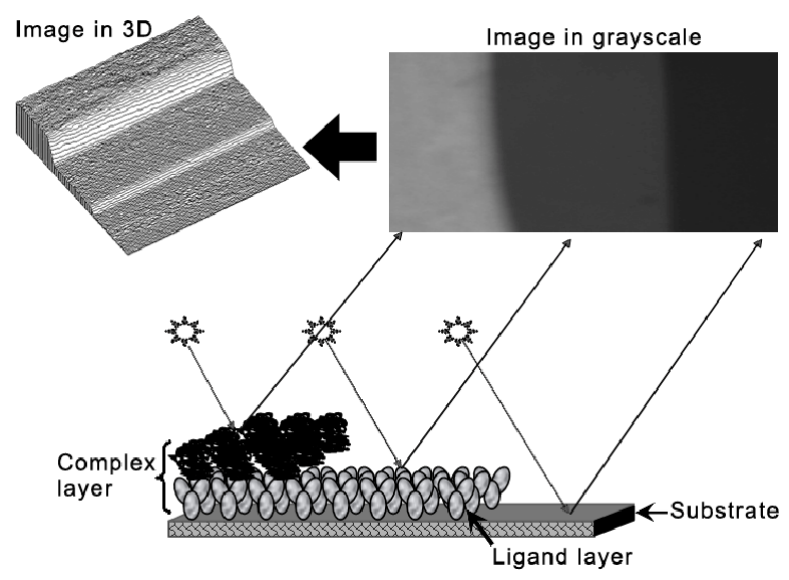

Figure 1 Model of bioprobe based on imaging ellipsometry [3]. The incident wave of polarized light as probe beam irradiates the sample and is modified, which makes the reflective beam carry sample information, such as protein surface concentration. The sample includes three parts: the bear substrate, the ligand layer and complex layer on the substrate, which could be seen in ellipsometric image with the value of reflection intensity I in grayscale. The variation of the protein surface concentration causes the value in grayscale of the image change. The surface concentration distribution of protein layer could be easily seen in three dimensions.

\section{GENERAL STRUCTURE OF THE BIOSENSOR AND EXPERIMENTAL SETUP}

Figure 2 gives the schematic diagram of a general structure of the biosensor based on imaging ellipsometry.

The biosensor is combined with several parts that are the control system, the microfluidic reactor array, the imaging ellipsometer as a reader and the biosensor database as well as software.

The control system has functions of hardware motion control and image data acquisition, played crucial role for performance and operation of the biosensor as an automatic system. A PC computer is used as the operation platform and data processor. Under the operation of homemade special software, a motion control card inserted in the computer produced motion signal for motor driver. The mechanical motion parts in microfluidic reactor array and imaging ellipsometer powered by the motor is driven automatically when needed. An image board inserted in the computer is used to transfer the image signal captured with imaging ellipsometer to a digital format.

The microfluidic reactor array is designed for protein array fabrication, solution delivery and biomolecule interaction. A microfluidic array containing many channels is used for fluid transferring to the sensing surface and forming a protein 
array [8]. Each channel has independent inlet and outlet, which can be used individually to deliver solutions to each cell of the array and covalently immobilize ligands on surface simultaneously. The fluid such as protein solution, purified water, rinsing fluid in each test tube is transferred to the microfluidic array by a peristaltic pump successively.

A protein array prepared by the microfluidic reactor is used for a parallel detection of protein interaction, and then, the protein microarray would be visualized by the imaging ellipsometer for protein detection. The imaging ellipsometer is based on a configuration of Polarizer-Compensator-Specimen-Analyzer (PCSA). The probe light beam with single wavelength is expended and collimated to ensure that each point in the view field of the protein array has the same angle of incidence. In order to carry out the quantitative measurement, off-null ellipsometry sampling method [2] is used due to its fast speed in the order of sub-second. The protein array is imaged by a lens into a $2 \mathrm{D}$ imaging sensor which transfers the image signal into computer and forms a digital image for further analysis. Under the operation of control system, the imaging ellipsometer system featured some automatic functions including the auto-focusing, the setting of polarizer, analyzer, compensator and the variation of the angle of incidence, etc.

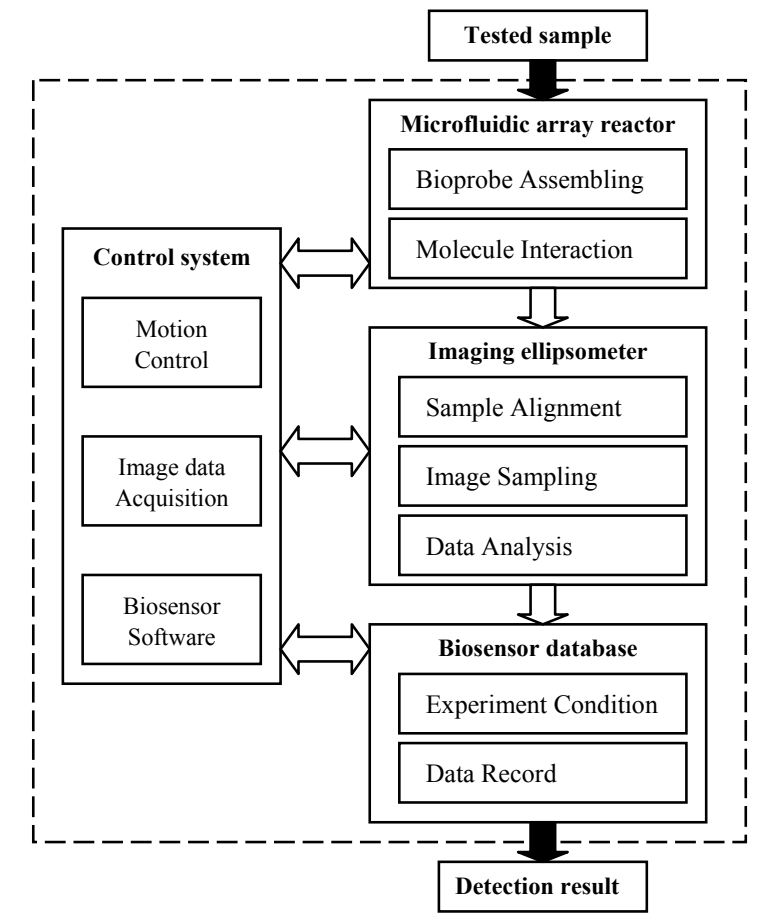

Figure 2 Schematic of Biosensor based on imaging ellipsometry [3]

The biosensor database is very important for the biosensor operation and applications. It has several functions: (1) Reference for experimental conditions setting from previous experiments; (2) Record and analysis of measurement conditions in microfluidic reactor array and imaging ellipsometer, such as measurement procedure and parameters, such as the fluid speed, reaction time, and polarizing components (polarizer, analyzer and compensator) setting, etc. (3) Record of the data of analyte, such as the bio-specificity, the molecule mass, concentrations, and corresponding ligands, (4) Record of detection condition, experiment data and analysis results, etc. All these are needed for users and potential applications. 


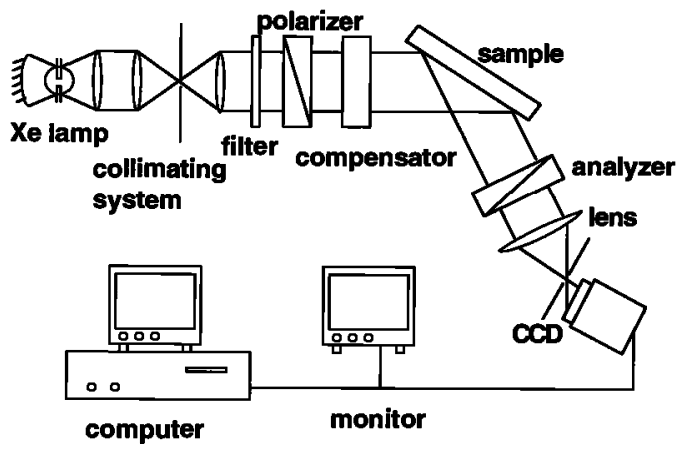

Figure 3 Schematic diagram of an imaging ellipsometer with an expanded beam, fixed polarizing components, and a CCD camera [1].

A general experimental set-up is shown in Figure 3 [1] It is based on a PCSA ellipsometer with a collimated expanded beam and a CCD camera as a detector. In the design of the optical system, special consideration should be taken of the following.

Source intensity and detector linearity: operation in both null and off-null ellipsometric modes is required. Null ellipsometry is independent of detector linearity and source intensity fluctuations. However, off-null ellipsometry is not and therefore the stability of the light source and the linearity of the sensor elements in the CCD camera is important.

Parallel light: The expanded probe beam must have parallel light to ensure that the angle of incidence is the same over the entire test surface. This is essential for the quantification of the surface thickness distribution.

Beam uniformity: The intensity over the cross section of the probe beam must be constant, because the intensity distribution over the beam area after reflection is a measure of the thickness distribution of the layer under study. A nonuniformity of the probe beam intensity leads to errors in the thickness distribution.

In the previous experimental setups [1,9], a $75 \mathrm{~W}$ xenon arc lamp and a collimating system are used as a light source to provide an expanded parallel probe beam with a diameter of $25 \mathrm{~mm}$. The xenon lamp is mounted in a standard housing (60010 lamp housing, ORIEL, U.S.A.) and driven by a stabilized power supply (68805 universal power supply, ORIEL, U.S.A.) under constant current conditions. The collimating system consists of an optical condenser with a short focal distance lens $(45 \mathrm{~mm})$ combined with a double convergent lens collimator with a pinhole. The former is set close to the lamp in order to collect as much light as possible. Its focal point is located in the emitting region of the lamp. The off- focal light and the light reflected from the bulb wall introduce a nonparallel part in the beam, which is eliminated with the double convergent lens collimator. The back focal plane of the first convergent lens is coincident with the front focal plane of the second convergent lens and the pinhole is located in the coincident plane of the two focal planes. When the quasi-parallel beam from the condenser passes through the first lens, the parallel part of the beam will focus at the center of the focal plane and the nonparallel part will focus in the off-center region. The pinhole blocks the off-center part. There is a trade-off between intensity on one hand and parallelism and uniformity on the other hand. A high parallelism and uniformity requires that the diameter of the pinhole should be as small as possible; however, a small diameter results in a low intensity. In our setup, a pinhole diameter of $0.6 \mathrm{~mm}$ is used, which results in a nonparallelism and non-uniformity of the probe beam of less than $0.3^{\circ}$ and $2 \%$, respectively.

To increase the ellipsometric contrast, a wavelength selective filter $(632.8 \mathrm{~nm})$ with a bandwidth of $10 \mathrm{~nm}$ is placed in the incident optical path. The light then passes through a dichroic polarizer and a compensator with a quarter-wave-plate at the wavelength of $632.8 \mathrm{~nm}$. The monochromatic and polarized beam is directed onto the sample at an angle of incidence of $76^{\circ}$. The reflected beam passes through another dichroic polarizer (called the analyzer) and a focusing lens. The lens projects the ellipsometric image of the sample on the sensor area of a CCD camera. The aperture of the lens should be larger than the illuminated region. In Figure 3 it is shown that the photosensitive chip of the CCD camera is set obliquely to the optical axis, in order to get a clear image of the entire sample surface. The reason for this is that the sample surface as an objective plane is located obliquely at the angle of incidence of the beam, and consequently the corresponding image plane is located non-perpendicularly to the optical axis of the system. The angle between the 
normal of the CCD chip and the optical axis depends on the incident angle of the probe beam and the objective distance, that is, the distance from the sample center to the imaging lens.

An achromatic lens is used to image the specimen onto a scientific B/W video CCD camera (SONY XC-ST30CE, Japan), with a spectral range of 400 to $900 \mathrm{~nm}$, sensitive area of $768 \times 576$ pixels on $6.0 \mathrm{~mm}$ x $4.96 \mathrm{~mm}$, and single pixel size of $6.5 \mu \mathrm{m} \times 6.25 \mu \mathrm{m}$. The electronic gain can be fixed for quantitative measurements, the output signal speed is 30 frames per second at maximum, and the nonlinearity of the CCD camera is better than $1 \%$. The image signal is transferred to an image board (Meter-II, Matrox, Canada) inserted in the computer for A/D converting. The ellipsometric images are recorded on an 8 bit (0 255) or 10 bit (0-1023) grayscale format. A monitor is connected to the video camera for sample positioning and null ellipsometry adjustments.

In normal operation, the compensator is fixed at an azimuth of $45^{\circ}$ to the plane of incidence. The analyzer and the polarizer are adjusted so that the ellipsometric null conditions are fulfilled on a bare silicon substrate. The absolute beam intensity and the gain of the CCD camera are not necessary to determine. It is sufficient to measure the relative intensity distribution over the beam after reflection to obtain the thickness distribution of a layer, as long as a reference sample with known layer thickness is used for an absolute calibration. Thickness calibration measurements are done with a null ellipsometer. The refractive index used for $\mathrm{SiO}_{2}$ is 1.457. The refractive index for proteins is in the range 1.48 -1.57 . Here we have used 1.5 throughout. Simple calculations using the exact ellipsometric expressions show that if the index is wrong, e.g. \pm 0.02 , the thickness error is less than $2 \%$.

\section{RECENT IMPROVEMENT}

Considering the dispersive effect of the protein microarray, the related experimental condition to the sensitivity and SNR, we have introduced a spectroscopic light source, optimization settings of polarization components [10] and a low noise CCD imaging [11].

We develops a spectroscopic imaging ellipsometry (SIE) $[9,12]$ system for the biosensor. It has the lateral resolution of optical imaging ellipsometry with various wavelengths. A 150-W Xenon short arc lamp (OSRAM, Germany) operated by a supply (Newport, U.S.) with light ripple less than $0.5 \%$ regulation accuracy is used to provide a stable outgoing flux with continuous spectrum from IR $(1700 \mathrm{~nm})$ to UV $(190 \mathrm{~nm})$. In order to adequately utilize the light flux, an achromatic convergent lens set with a focal length of $200 \mathrm{~mm}$ and a diameter of $50 \mathrm{~mm}$ is used to converge the light beam into the entrance slit of a mechanical scanning grating monochromator (7-Star, China) with a focal length of $150 \mathrm{~mm}$ and an f-number of $1 / 4.5$. The monochromator controlled by a computer provides a spectrum of 260-1600 nm with adjustable entrance and an exit for the control of light intensity and bandwidth, respectively. A glass optical fiber bound (Chunhui, China) with a spectrum of 380-1300 nm and a diameter of $1.0 \mathrm{~mm}$ is mounted flexibly to transfer the incident light from the exit of the monochromator to the focus point of the second achromatic convergent lens set. It collimates the single wavelength light into an expanded parallel beam with a diameter of $25 \mathrm{~mm}$ for imaging ellipsometry at an angle of incidence.

Two Glan-Taylor prisms (MELLES GRIOT, USA) with a spectral range of 250-2300 nm and an extinction ratio of 105 are used as the polarizer and the analyzer, respectively. A mica retardation plate (MELLES GRIOT, USA) with a spectral range of $400-700 \mathrm{~nm}$ is used as a compensator. Its phase retardation between fast and slow axis is near $90^{\circ}$, which is calibrated by a commercial V-VASE ellipsometer (J.A.Woollam, USA) and fitted by the Cauchy model. In order to adjust the azimuth with respect to the plane of incidence, the polarizer, compensator, and analyzer are fixed in hollow annular rotation stages driven by a servo motor (Newport, US) with a maximum speed of $40 \% \mathrm{~s}$ and an absolute accuracy of $0.015^{\circ}$. An achromatic imaging lens set (Zeiss, Germany) with a focal length of $50 \mathrm{~mm}, \mathrm{f}-1.4$ is used to image the specimen onto a cool CCD camera (Andor Technology, DU937N-BV, read noise 10.3e@2.5 MHz, dark current $0.0005 \mathrm{e}-/ \mathrm{pixel} / \mathrm{sec} @-80^{\circ} \mathrm{C}$ ). The camera features a corresponding spectrum range of $400-900 \mathrm{~nm}$, $512 \times 512$ active pixels, $13 \times 13 \mu \mathrm{m}$ pixel size, $6.6 \times 6.6 \mathrm{~mm}$ image area and boasts $95 \%$ QEmax., high dynamic range and exceptionally low readout noise. It benefits from negligible dark current with thermoelectric cooling down to $-100^{\circ} \mathrm{C}$, enabling use of significantly longer exposure times. The image signal is inputted into an image grab board (Matrox, Canada) inserted in the computer for analog-to-digital (A/D) conversion and the digital image processing and storage. The ellipsometric images are recorded in 14-bit grayscale format. Image averaging method available is used to improve the signal-to-noise ratio [13]. 
The improvement in imaging system brings an obvious improvement in the sensitivity and SNR. It makes specifications of the biosensor more fitted to practical requirements, the sensitivity for immunoglobulin molecule in $1 \mathrm{ng} / \mathrm{mL}$ and the time resolution of $0.04 \mathrm{~s}$, the consumption of ligand in $\mathrm{ng}$ and specimen in the order of $10 \mu \mathrm{l}$, the throughput of 48 independent channels, as well as a continues variable spectrum in visible region. For a protein pattern on a gold layer/glass substrate, the imaging resolution corresponding to the sensitivity increases about 4 times when the wavelength is chosen from $550 \mathrm{~nm}$ to $600 \mathrm{~nm}$, which shows the various spectroscopic light source is helpful for the biosensor data acquisition. Comparing with the previous system with the CCD of Sony XC-30CE used in TIRIE [4], the sensitivity has increased about one order and the detection limit for Hepatitis B surface antigen (HBsAg) 50 times better than the previous one. The main contribution to the improvement of the sensitivity and the detection limit comes from the use of the cool CCD [11].

\section{BIOMEDICAL APPLICATIONS}

The biosensor technique is developed mainly to focus on biomedical applications, and it has been successfully used for the biomolecule interaction [6,7], the cancer marker test[14], the clinic diagnosis for hepatitis B [15], the quantitative protein competitive adsorption [16], the kinetic detection for multi-protein interaction process [4, 11], and the virus detection [17, 18], etc. These biomedical applications just show some examples of the biosensor based on imaging ellipsometry in biomedical fields.

\section{CONCLUSION}

The improvement of the imaging system has shown a better sensitivity and SNR in the biosensor, and proved one order better than the previous one. It is helpful for protein interaction process detection in real-time. In this way, the high sensitivity makes it available of fast detection and protein weak interaction detection.

\section{ACKNOWLEDGEMENT}

The author gratefully acknowledges supports from National Basic Research Program of China 2009CB320300, National High Technology Research and Development Program of China 2008AA02Z419 and Chinese Academy of Sciences (KJCX2-YW-Mo3 and -M04) as well as K.C.Wong Education Foundation, Hong Kong, NNSFC 20805053.

\section{REFERENCES}

[1] G. Jin, P. Tengvall, I. Lundstrom, and H. Arwin, "A Bionsensor concept based on imaging ellipsometry for visualization of biomolecular interactions," Analytical Biochemistry 232 (1), 69-72 (1995).

[2] G. Jin, R. Jansson, and H. Arwin, "Imaging ellipsometry revisited: Developments for visualization of thin transparent layers on silicon substrates," Review of Scientific Instruments 67 (8), 2930-2936 (1996).

[3] G. Jin, "Development of biosensor based on imaging ellipsometry," Physica Status Solidi a-Applications and Materials Science 205 (4), 810-816 (2008).

[4] Y. Y. Chen, Z. H. Wang, Y. H. Meng, and G. Jin, "Biosensor with total internal reflection imaging ellipsometry," International Journal of Nanotechnology 4 (1-2), 171-178 (2007).

[5] G. Jin, Z. H. Wang, Y. H. Meng, P. Q. Ying, and L. H. Xia, "Optical proteinchip as microarrays for protein interaction determination," Proceedings of the 23rd Annual International Conference of the Ieee Engineering in Medicine and Biology Society, 23 2907-2910 (2001).

[6] G. Jin and Z. H. Wang, "Micro-systems for optical protein-chip," International Journal of Nonlinear Sciences and Numerical Simulation 3 (3-4), 191-194 (2002). 
[7] Z. H. Wang and G. Jin, "A label-free multisensing immunosensor based on imaging ellipsometry," Analytical Chemistry 75 (22), 6119-6123 (2003).

[8] Z. H. Wang, Y. H. Meng, P. Q. Ying, C. Qi, and G. Jin, "A label-free protein microfluidic array for parallel immunoassays," Electrophoresis 27 (20), 4078-4085 (2006).

[9] Y. H. Meng, Y. Y. Chen, C. Qi, L. Liu, and G. Jin, "An automatic imaging spectroscopic ellipsometer for characterization of nano-film pattern on solid substrate," Physica Status Solidi C - Current Topics in Solid State Physics, 5 (5), 1050-1053 (2008).

[10] L. Liu, Y. Niu, S. Chen, Y. H. Meng, H. W. Ma, and G. Jin, "Optimization of evanescent wave imaging for the visualization of protein adsorption layers," Science China-Physics Mechanics \& Astronomy 53 (10), 1805-1810 (2010).

[11] L. Liu, Y. Y. Chen, Y. H. Meng, S. Chen, and G. Jin, "Improvement for sensitivity of biosensor with total internal reflection imaging ellipsometry (TIRIE)," Thin Solid Films, In press (2011).

[12] Y. H. Meng and G. Jin, "Rotating compensator sampling for spectroscopic imaging ellipsometry," Thin Solid Films, In press (2011).

[13] Y. H. Meng and G. Jin, "Technique of grabbing and processing in ellipsometric imaging system," Optics and Precision Engineering 8 316-320 (2000).

[14] H. G. Zhang, C. Qi, Z. H. Wang, G. Jin, and R. J. Xiu, "Evaluation of a new CA15-3 protein assay method: Optical protein-chip system for clinical application," Clinical Chemistry 51 (6), 1038-1040 (2005).

[15] C. Qi, W. Zhu, Y. Niu, H. G. Zhang, G. Y. Zhu, Y. H. Meng, S. Chen, and G. Jin, "Detection of hepatitis B virus markers using a biosensor based on imaging ellipsometry," Journal of Viral Hepatitis 16 (11), 822-832 (2009).

[16] P. Q. Ying, G. Jin, and Z. L. Tao, "Competitive adsorption of collagen and bovine serum albumin - effect of the surface wettability," Colloids and Surfaces B-Biointerfaces 33 (3-4), 259-263 (2004).

[17] C. Qi, Y. Lin, J. Feng, Z. H. Wang, C. F. Zhu, Y. H. Meng, X. Y. Yan, L. J. Wan, and G. Jin, "Phage M13KO7 detection with biosensor based on imaging ellipsometry and AFM microscopic confirmation," Virus Research 140 (1-2), 79-84 (2009).

[18] C. Qi, X. S. Tian, S. Chen, J. H. Yan, Z. Cao, K. G. Tian, G. F. Gao, and G. Jin, "Detection of avian influenza virus subtype H5 using a biosensor based on imaging ellipsometry," Biosensors \& Bioelectronics 25 (6), 1530 $1534(2010)$. 